GLASNIK MATEMATIČKI

Vol. 39(59)(2004), $145-154$

\title{
ON DIRECTLY ADDITIVE SELECTORS FOR CONVEX AND STAR BODIES
}

\author{
MARIA MOSZYŃSKA \\ Warsaw University, Poland
}

\begin{abstract}
The paper deals with direct additivity of selectors, as well for convex as for star bodies. Sections 3 and 4 concern selectors for convex bodies. In Section 5 two methods of extending a selector for convex bodies to a selector for star bodies are considered, both preserving direct additivity.
\end{abstract}

\section{INTRODUCTION}

As it was proved by Schneider in 1971 ([10]), among all the selectors for compact convex subsets of $\mathrm{R}^{n}$ which are continuous with respect to the Hausdorff metric and equivariant under the isometries, only the Steiner point map is Minkowski additive.

We are interested in direct additivity of selectors, that is, roughly speaking, additivity with respect to direct sum.

In Sections 3 we give three examples of directly additive selectors for convex bodies: the Steiner point, the gravity centre, and the Čebyšev point. Section 4 concerns $G$-pseudo-centres, which are studied separately because in this case the situation is more complicated.

In Section 5 we consider two methods of extending selectors for convex bodies to those for star bodies.

Our motivation for studying selectors for star bodies is the following (compare [7], p.132). Various constructions for star bodies are defined in terms of radial function, which depends on the position of 0 in a star set considered. For instance, the intersection body $I A$, whose radial function is defined by

2000 Mathematics Subject Classification. 52A20, 52A30, 52A99.

Key words and phrases. Convex bodies, star bodies, selectors, direct sum. 
the formula

$$
\varrho_{I A}(u):=V_{n-1}\left(A \cap u^{\perp}\right)
$$

for $u \in S^{n-1}$, (see [3]), depends on the choice of the origin. Let us recall that the notion of intersection body plays an essential role in geometric tomography (see [5]).

\section{Preliminaries}

We follow, in principle, terminology and notation used in [12]. In particular, bd, int, cl, conv, lin, and aff are, respectively, boundary, interior, closure, convex hull, linear hull, and affine hull. For $v \in \mathrm{R}^{n} \backslash\{0\}$,

$$
\operatorname{pos} v:=\{\lambda v \mid \lambda \geq 0\} .
$$

The $k$-dimensional Lebesgue measure in Euclidean $k$-dimensional space is $\lambda_{k}$, the $k$-dimensional spherical measure in a $k$-dimensional unit sphere is $\sigma_{k}$ (or simply $\sigma$, if it does not lead to a confusion). The $k$-dimensional Hausdorff measure is $\mathcal{H}^{k}$.

$B^{n}$ and $S^{n-1}$ are the unit ball and the unit sphere in $\mathrm{R}^{n}$, respectively, and $\mathcal{K}^{n}$ is the class of compact convex subsets of $\mathrm{R}^{n}$. However, a convex body in $\mathrm{R}^{n}$ is assumed to have the non-empty interior, while Schneider in [12] refers to any compact convex set as a convex body; $\mathcal{K}_{0}^{n}$ is the class of convex bodies in $\mathrm{R}^{n}$. More generally, for any Euclidean space $E$, the class of all compact convex subsets of $E$ and the class of all convex bodies in $E$ are $\mathcal{K}(E)$ and $\mathcal{K}_{0}(E)$, respectively.

For any $A \in \mathcal{K}^{n}$, the intrinsic volume of order $i$ of $A$ is $V_{i}(A)$. Let us recall that $V_{0}(A)=1$ for every $A$.

Let $\mathcal{F} \subset \mathcal{K}^{n}$. A map $s: \mathcal{F} \rightarrow \mathrm{R}^{n}$ is called a selector for $\mathcal{F}$ if $s(A) \in A$ for every $A \in \mathcal{F}$.

We shall also consider selectors for star sets. Let $A \subset \mathrm{R}^{n}$. The kernel of $A$ is defined by the formula

$$
\operatorname{ker} A:=\{a \in A \mid \forall x \in A \Delta(a, x) \subset A\},
$$

where $\Delta(a, x)$ is the segment with endpoints $a, x$.

A subset $A$ of $\mathrm{R}^{n}$ is called a star set if $\operatorname{ker} A \neq \emptyset$; a compact star set $A$ in $\mathrm{R}^{n}$ is called a star body if $\mathrm{cl} \operatorname{int} A=A$.

Let $\mathcal{S}^{n}$ be the class of compact star sets in $\mathrm{R}^{n}$ and $\mathcal{S}_{0}^{n}$ the class of compact star bodies in $\mathrm{R}^{n}$. Similarly as we do for convex bodies, we use the symbols $\mathcal{S}(E)$ and $\mathcal{S}_{0}(E)$ for the classes of suitable star sets in any Euclidean space $E$.

Let $\mathcal{F} \subset \mathcal{S}^{n}$. A map $s: \mathcal{F} \rightarrow \mathrm{R}^{n}$ is a selector for $\mathcal{F}$ if and only if $s(A) \in \operatorname{ker} A$ for every $A \in \mathcal{F}$ (see [7]).

A selector $s: \mathcal{F} \rightarrow \mathrm{R}^{n}$ is said to be equivariant under an isometry $f$ of $\mathrm{R}^{n}$ onto itself if for every $A \in \mathcal{F}$

$$
s(f(A))=f(s(A)) .
$$


For any $m \leq n$, let $\mathcal{E}_{m}^{n}$ be the family of $m$-dimensional affine subspaces of $\mathrm{R}^{n}$, and let

$$
\mathcal{E}^{n}:=\bigcup_{m \leq n} \mathcal{E}_{m}^{n}
$$

Lemma 2.1. Let $E \in \mathcal{E}_{m}^{n}$ for some $m \leq n$ and let $f_{1}, f_{2}: E \rightarrow \mathrm{R}^{m}$ be isometries. If $A \in \mathcal{K}_{0}(E)$ and a selector $s: \mathcal{K}_{0}^{m} \rightarrow \mathrm{R}^{m}$ is equivariant under the isometries of $\mathrm{R}^{m}$, then

$$
f_{1}^{-1}\left(s\left(f_{1}(A)\right)\right)=f_{2}^{-1}\left(s\left(f_{2}(A)\right)\right) .
$$

Proof. Let $f:=f_{2} \circ f_{1}^{-1}$. Then $f$ is an isometry of $\mathrm{R}^{m}$ mapping $f_{1}(A)$ on $f_{2}(A)$. Thus, by $(2.1)$,

$$
f\left(s\left(f_{1}(A)\right)\right)=s\left(f_{2}(A)\right),
$$

which is equivalent to $(2.2)$.

Let now $\oplus$ be the direct sum: if $\mathrm{R}^{n}=E_{1} \oplus E_{2}$ for two affine subspaces $E_{1}, E_{2}$ of $\mathrm{R}^{n}$ with positive dimensions, then for every $X_{i} \subset E_{i}, i=1,2$,

$$
X_{1} \oplus X_{2}:=X_{1}+X_{2} .
$$

Thus, direct sum operation is a restriction of the Minkowski addition.

For any $A \in \mathcal{K}^{n}$, let $\xi_{A}: \mathrm{R}^{n} \rightarrow A$ be the metric projection (i.e., the nearest point map) of $\mathrm{R}^{n}$ on $A$. We shall need the following property of this map.

Lemma 2.2. If $\mathrm{R}^{n}=E_{1} \oplus E_{2}$ for $E_{1}, E_{2}$ orthogonal, $a_{i} \in E_{i}$, and $X_{i} \in$ $\mathcal{K}\left(E_{i}\right)$ for $i=1,2$, then

$$
\xi_{X_{1} \oplus X_{2}}\left(a_{1}+a_{2}\right)=\xi_{X_{1}}\left(a_{1}\right)+\xi_{X_{2}}\left(a_{2}\right) .
$$

Proof. Let $a=a_{1}+a_{2}$. Then

$$
\begin{aligned}
& \inf \left\{\|x-a\| \mid x \in X_{1} \oplus X_{2}\right\}= \\
& \quad=\inf \left\{\sqrt{\left\|x_{1}-a_{1}\right\|^{2}+\left\|x_{2}-a_{2}\right\|^{2}} \mid x_{i} \in X_{i}, \quad i=1,2\right\} .
\end{aligned}
$$

Thus, if $x_{i} \in E_{i}$ and $x=x_{1}+x_{2}$, then $x=\xi_{X_{1} \oplus X_{2}}\left(a_{1}+a_{2}\right)$ if and only if $x_{i}=\xi_{X_{i}}\left(a_{i}\right)$ for $i=1,2$. This completes the proof.

\section{DiRectly ADditive SELECTORS FOR CONVEX Bodies}

We begin with selectors for $\mathcal{K}^{n}$. For our purpose it is reasonable to consider a sequence of selectors, $\left(s^{(m)}: \mathcal{K}^{m} \rightarrow \mathrm{R}^{m}\right)_{m \leq n}$ instead of an individual selector for a fixed $n$.

In view of Lemma 2.1, for a given $n$, every sequence $\left(s^{(m)}: \mathcal{K}^{m} \rightarrow\right.$ $\left.\mathcal{R}^{m}\right)_{m \leq n}$ of equivariant selectors determines the family of selectors,

$$
\mathbf{s}:=\left(s_{E}: \mathcal{K}(E) \rightarrow E\right)_{E \in \mathcal{E}^{n}},
$$


defined as follows: for every $E \in \mathcal{E}_{m}^{n}$ and $A \in \mathcal{K}(E)$,

$$
s_{E}(A):=f^{-1} s^{(m)} f(A),
$$

where $f: E \rightarrow \mathrm{R}^{m}$ is an isometry.

The following is evident.

Proposition 3.1. Let $\mathbf{s}$ be the family determined by a sequence of equivariant selectors, defined by (3.1). If $E, E^{\prime} \in \mathcal{E}_{m}^{n}$ for some $m \leq n$, then for every isometry $f: E \rightarrow E^{\prime}$ and $A \in \mathcal{K}(E)$

$$
f\left(s_{E}(A)\right)=s_{E^{\prime}}(f(A)) .
$$

In particular, every member of the family $\mathbf{s}$ is equivariant under the isometries of the corresponding affine subspace.

Let us consider three examples:

For $A \in \mathcal{K}_{0}^{n}$, let $c^{(n)}(A)$ be the centroid of $A$ (i.e. the gravity centre with respect to $\left.\lambda_{n}\right)$ :

$$
c^{(n)}(A):=\frac{1}{\lambda_{n}(A)} \int_{A} x d \lambda_{n}(x) .
$$

Further, for any $A \in \mathcal{K}^{n}$, let $p^{(n)}(A)$ and $\check{c}^{(n)}(A)$ be, respectively, the Steiner point and the Čebyšev point of $A$ :

$$
p^{(n)}(A):=\frac{1}{\kappa_{n}} \int_{S^{n-1}} u h_{A}(u) d \sigma(u),
$$

and $\check{c}^{(n)}(A)$ is the centre of the smallest ball in $\mathrm{R}^{n}$ containing $A$.

As is well known, all these selectors are equivariant under the isometries.

For $E \in \mathcal{E}^{n}$ the points $c_{E}(A), p_{E}(A)$, and $\check{c}_{E}(A)$ are the centroid, the Steiner point, and the $\check{C}$ ebyšev point of $A$ with respect to $E$.

We now define direct additivity as follows.

DEFINITION 3.2. Let $\mathbf{s}=\left(s_{E}\right)_{E \in \mathcal{E}^{n}}$ be the family determined by a sequence $\left(s^{(m)}\right)_{m \leq n}$ of equivariant selectors (see (3.1)). The family $\mathbf{s}$ is directly additive (or, the sequence $\left(s^{(m)}\right)_{m \leq n}$ is directly additive) if and only if

$$
s^{(n)}\left(A_{1} \oplus A_{2}\right)=s_{E_{1}}\left(A_{1}\right)+s_{E_{2}}\left(A_{2}\right)
$$

whenever $E_{i}=$ aff $A_{i}$ and $\mathrm{R}^{n}=E_{1} \oplus E_{2}$ with $E_{1}, E_{2}$ orthogonal.

TheOREm 3.3. The family of centroids, c, of the Steiner point maps, $\mathbf{p}$,

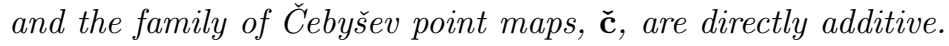

PROOF. Since the Lebegue measure is multiplicative, that is, for every pair of orthogonal subspaces $E_{1}, E_{2}$ of $\mathrm{R}^{n}$ with $\operatorname{dim} E_{i}=n_{i}$ and every Borel sets $X_{1}, X_{2}$ with $X_{i} \subset E_{i}$

$$
\lambda_{n}\left(X_{1} \oplus X_{2}\right)=\lambda_{n_{1}}\left(X_{1}\right) \cdot \lambda_{n_{2}}\left(X_{2}\right),
$$

from (3.2) it follows that $\mathbf{c}$ is directly additive. 
Since the Steiner point is independent of dimension of the ambient space, that is, for every subspace $E$ of $\mathrm{R}^{n}$ with $\operatorname{dim} E<n$ and every $A \in \mathcal{K}(E)$

$$
p_{E}(A)=p^{(n)}(A),
$$

the Minkowski additivity of $p$ implies direct additivity of $\mathbf{p}$.

It remains to prove direct additivity of $\check{\mathbf{c}}$.

Let $\mathrm{R}^{n}=E_{1} \oplus E_{2}$ for some orthogonal affine subspaces $E_{1}, E_{2}$ with $\operatorname{dim} E_{i} \geq 1$; let aff $A_{i}=E_{i}$, and $A_{i} \in \mathcal{K}_{0}\left(E_{i}\right)$ for $\mathrm{i}=1,2$.

Let $a_{i}=\check{c}_{E_{i}}\left(A_{i}\right)$, and let $B\left(a_{i}, r_{i}\right)$ be the Čebyšev ball of $A_{i}$ in $E_{i}$ for $i=1,2$ (i.e. the smallest ball in $E_{i}$ containing $A_{i}$ ).

Let $X:=B\left(a_{1}, r_{1}\right) \oplus B\left(a_{2}, r_{2}\right)$; then $A_{1} \oplus A_{2} \subset X$. Let $a=a_{1}+a_{2}$. Since, evidently, $a$ is the symmetry centre of $X$, it follows that $a=\check{c}^{(n)}(X)$.

Let $\check{B}$ be the Čebyšev ball of $X$. Then $\check{B}=B(a, r)$, where

$$
r=\sqrt{\left(r_{1}\right)^{2}+\left(r_{2}\right)^{2}} .
$$

Suppose that $\check{B}$ is not the Čebyšev ball of $A_{1} \oplus A_{2}$. Thus, there exists a ball $B_{0}$ containing $A_{1} \oplus A_{2}$, with a radius $r_{0}<r$. Let $B_{0, i}:=B_{0} \cap E_{i}$ for $i=1,2$. Then the ball $B_{0, i}$ in $E_{i}$ contains $A_{i}$, whence its radius $r_{0, i}$ is at least $r_{i}$. But

$$
r_{0}=\sqrt{\left(r_{0,1}\right)^{2}+\left(r_{0,2}\right)^{2}}<r,
$$

a contradiction. Hence $\check{B}$ is the Čebyšev ball of $A_{1} \oplus A_{2}$, and thus $a=$ $\check{c}^{(n)}\left(A_{1} \oplus A_{2}\right)$, i.e.,

$$
\check{c}^{(n)}\left(A_{1} \oplus A_{2}\right)=\check{c}_{E_{1}}\left(A_{1}\right)+\check{c}_{E_{2}}\left(A_{2}\right) .
$$

Let us notice that direct additivity of $\mathbf{p}$ is a particular case of much more general fact (Theorem 3.5).

Let us recall that every $A \in \mathcal{K}^{n}$ determines a sequence of curvature measures $\Phi_{k}(A, \cdot), k=0, \ldots, n$ (see [11]). This notion was originally introduced and studied by H. Federer in [2] for arbitrary set $A$ with positive reach in $\mathrm{R}^{n}$; we shall need the following version of his Theorem 5.14 (4) in [2] p. 422.

Theorem 3.4. Let $A_{i} \in \mathcal{K}_{0}\left(E_{i}\right)$ and $A_{i} \subset E_{i}$ for orthogonal affine subspaces $E_{1}, E_{2}$ of $\mathrm{R}^{n}$, with $\operatorname{dim} E_{i}=n_{i}$, where $n_{1}+n_{2}=n$, and let $k \in\{0, \ldots, n\}$. Then for every Borel sets $X_{i}$ in $E_{i}, i=1,2$,

$$
\Phi_{k}\left(A_{1} \oplus A_{2}, X_{1} \oplus X_{2}\right)=\sum_{i_{1}+i_{2}=k} \Phi_{i_{1}}\left(A_{1}, X_{1}\right) \cdot \Phi_{i_{2}}\left(A_{2}, X_{2}\right) .
$$

Let us consider the curvature centroids, i.e. the gravity centres with respect to curvature measures:

$$
p_{i}(A):=c_{\Phi_{n-i}(A, \cdot)}(A),
$$


In particular, $p_{n}$ is the Steiner point map, i.e., $p_{n}=p^{(n)}$. (see [12]). As a consequence of the Federer Theorem 3.4, we obtain the following result concerning a relationship between the selectors $p_{0}, \ldots, p_{n}$.

THEOREM 3.5. Let $E_{1}, E_{2}$ be two orthogonal affine subspaces of $\mathrm{R}^{n}$ with $\operatorname{dim} E_{k}=n_{k}$ and $n_{1}+n_{2}=n$. Let $A_{1}, A_{2} \in \mathcal{K}^{n}$ and $A_{k} \subset E_{k}$. Then, for every $i \in\{0, \ldots, n\}$

$$
p_{n-i}\left(A_{1} \oplus A_{2}\right)=\sum_{i_{1}+i_{2}=i} \alpha_{i_{1}, i_{2}} \cdot\left(\left(p_{n_{1}-i_{1}}\right)_{E_{1}}\left(A_{1}\right)+\left(p_{n_{2}-i_{2}}\right)_{E_{2}}\left(A_{2}\right)\right),
$$

where, for $i_{k} \leq n_{k}, k=1,2$,

$$
\alpha_{i_{1}, i_{2}}:=\frac{V_{i_{1}}\left(A_{1}\right) V_{i_{2}}\left(A_{2}\right)}{V_{i}\left(A_{1} \oplus A_{2}\right)} .
$$

Proof. By Theorem 3.4 combined with the Fubini theorem,

$$
\begin{gathered}
\int_{A_{1} \oplus A_{2}} x d \Phi_{i}\left(A_{1} \oplus A_{2}, x\right)=\sum_{i_{1}+i_{2}=i} \int_{A_{1}} \int_{A_{2}}\left(x_{1}+x_{2}\right) d \Phi_{i_{1}}\left(A_{1}, x_{1}\right) d \Phi_{i_{2}}\left(A_{2}, x_{2}\right) \\
=\sum_{i_{1}+i_{2}=i} V_{i_{1}}\left(A_{1}\right) V_{i_{2}}\left(A_{2}\right)\left(\left(p_{n_{1}-i_{1}}\right)_{E_{1}}\left(A_{1}\right)+\left(p_{n_{2}-i_{2}}\right)_{E_{2}}\left(A_{2}\right)\right) .
\end{gathered}
$$

To complete the proof, it now remains to use (3.7) and (3.8).

Finally, let us mention that direct additivity of centre of the minimal ring was studied in [6]; it was proved that this selector was not directly additive.

\section{Problem of direct additivity for $G$-PSeudo-Centres}

In [8], for any subgroup $G$ of $O(n)$, the notion of $G$-pseudo-centre of a convex body in $\mathrm{R}^{n}$ was defined:

for $A \in \mathcal{K}_{0}^{n}$, the set $P_{G}(A)$ of $G$-pseudo-centres of $A$ consists of maximizers of the function

$$
A \ni x \mapsto V_{n}\left(\bigcap_{g \in G} g(A-x)\right) .
$$

Generally, the set $P_{G}(A)$ does not have to be a singleton. However, if $G$ is generated by the central symmetry $\sigma_{0}^{n}$ of $\mathrm{R}^{n}$, then every $A$ has a unique $G$-pseudo-centre, $p_{G}(A)$ (compare [1]) ${ }^{1}$; moreover, $\left\langle\sigma_{0}^{n}\right\rangle$ is the only group with this property.

Further, for any $G \subset O(n)$, if fix $G=\{0\}$, then every strictly convex $A$ has a unique $G$-pseudo-centre (see $[8,9]$ ).

We start with the following

Definition 4.1. Let $\left(E_{1}, E_{2}\right)$ be a pair of orthogonal linear subspaces of $\mathrm{R}^{n}$, with $\mathrm{R}^{n}=E_{1} \oplus E_{2}$, and let $G_{E_{i}}$ be a group of linear isometries of $E_{i}$ for $i=1,2$.

\footnotetext{
${ }^{1}$ In [1] this point is called quasi-centre of $A$
} 
(i) For every $g_{i} \in G_{i}, i=1,2$, the map $g_{1} \star g_{2}: \mathrm{R}^{n} \rightarrow \mathrm{R}^{n}$ is defined by

$$
\left(g_{1} \star g_{2}\right)\left(x_{1}+x_{2}\right):=g_{1}\left(x_{1}\right)+g_{2}\left(x_{2}\right) \text { for every } x_{i} \in E_{i}, i=1,2 .
$$

(ii) $G_{E_{1}} \star G_{E_{2}}:=\left\{g_{1} \star g_{2} \mid g_{i} \in G_{E_{i}}\right\}$.

REMARK 4.2. Under the assumptions of 4.1, the group $G_{E_{1}} \star G_{E_{2}}$ is isomorphic to the Cartesian product of these two groups.

We shall now prove the following (compare 3.7 in [8]).

THEOREM 4.3. Let $E_{1}, E_{2}$ be orthogonal linear subspaces of $\mathrm{R}^{n}$ with $\operatorname{dim} E_{i}=n_{i}$ and $n_{1}+n_{2}=n$, and let $G_{i}$ be a group of linear isometries of $E_{i}$. Then, for every $A_{i} \in \mathcal{K}\left(E_{i}\right), i=1,2$,

$$
P_{G_{1} \star G_{2}}\left(A_{1} \oplus A_{2}\right)=P_{G_{1}}\left(A_{1}\right) \oplus P_{G_{2}}\left(A_{2}\right) .
$$

If, in particular, $P_{G_{i}}\left(A_{i}\right)$ is a singleton for $i=1,2$, then $P_{G_{1} \star G_{2}}\left(A_{1} \oplus A_{2}\right)$ is a singleton too, and

$$
p_{G_{1} \star G_{2}}\left(A_{1} \oplus A_{2}\right)=p_{G_{1}}\left(A_{1}\right)+p_{G_{2}}\left(A_{2}\right) .
$$

Proof. Let $A=A_{1} \oplus A_{2}$ and let $x \in A$. Then $x=x_{1}+x_{2}$ for some $x_{i} \in A_{i}, i=1,2$, and

$$
\begin{aligned}
\bigcap_{g \in G_{1} \star G_{2}} g(A-x) & =\bigcap_{g_{1} \in G_{1}} \bigcap_{g_{2} \in G_{2}} g_{1}\left(A_{1}-x_{1}\right) \oplus g_{2}\left(A_{2}-x_{2}\right) \\
& =\left(\bigcap_{g_{1} \in G_{1}} g_{1}\left(A_{1}-x_{1}\right)\right) \oplus\left(\bigcap_{g_{2} \in G_{2}} g_{2}\left(A_{2}-x_{2}\right)\right) .
\end{aligned}
$$

Thus

$$
x \in P_{G_{1} \star G_{2}}(A) \Longleftrightarrow x_{i} \in P_{G_{i}}\left(A_{i}\right) \text { for } i=1,2 \text {. }
$$

Let us notice that the property of $G$-pseudo-centres described by Theorem 4.3 is weaker than direct additivity, because $G_{i}$ depends on $E_{i}$. To define the suitable family $\mathbf{p}_{\mathbf{G}}$ of selectors for all the $\mathcal{E}^{n}$, we need equivariance with respect to the isometries (see Lemma 1.1). As was proved in [9] (see (2.2) in Example 2.2 and Example 2.5), $G$-pseudo-centre $p_{G}$ is equivariant with respect to all isometries if and only if $G=<\sigma_{0}>$.

Corollary 4.4. The sequence $\left(p_{G_{n}}: \mathcal{K}^{n} \rightarrow \mathrm{R}^{n}\right)_{n \in \mathrm{N}}$ of $G_{n}$-pseudocentres induces the family $\mathbf{p}_{\mathbf{G}}$ if and only if $G_{n}$ is generated by $\sigma_{0}^{n}$ for every $n$. This unique family is directly additive. 


\section{EXTENSIONS OF SELECTORS FROM CONVEX TO STAR BODIES}

Let us recall two methods of extending selectors for $\mathcal{K}_{0}^{n}$ to selectors for $\mathcal{S}_{0}^{n}$ (compare [7]). The first method can be applied to an arbitrary selector $s: \mathcal{K}_{0}^{n} \rightarrow \mathrm{R}^{n}$ : its extension $\bar{s}: \mathcal{S}_{0}^{n} \rightarrow \mathrm{R}^{n}$ is defined by the formula

$$
\bar{s}(A):=\xi_{\operatorname{ker} A}(s(\operatorname{conv} A)) \text { for every } A \in \mathcal{S}_{0}^{n} .
$$

The second method can be applied to a selector $s$ which satisfies the following condition:

(*) There exists a family $\left(\Phi_{A}: A \rightarrow \mathrm{R}\right)_{A \in \mathcal{K}^{n}}$ of functions such that

(i) for every $A \in \mathcal{K}_{0}^{n}$, the point $s(A)$ is the unique maximizer (minimizer) of $\Phi_{A}$,

(ii) for every $A \in \mathcal{K}_{0}^{n}$ and $X \in \mathcal{K}^{n}$ with $X \subset A$, the function $\Phi_{A} \mid X$ has the unique maximizer (minimizer), $s(A, X)$.

If $s$ satisfies $\left({ }^{*}\right)$, then its extension $\tilde{s}: \mathcal{S}_{0}^{n} \rightarrow \mathrm{R}^{n}$ is defined by the formula

$$
\tilde{s}(A):=s(\operatorname{conv} A, \operatorname{ker} A) \text {. }
$$

In [7] the second method was introduced ${ }^{2}$ for $s$ being the radial centre map $r_{\phi}: \mathcal{K}_{0}^{n} \rightarrow \mathrm{R}^{n}$ associated with a function $\phi: \mathrm{R}_{+} \rightarrow \mathrm{R}_{+}$. If $n \geq 2$, the function $\Phi_{A}$ for which $r_{\phi}(A)$ is the maximizer is then defined by

$$
\Phi_{A}(x):=\int_{S^{n-1}} \phi \varrho_{A-x}(u) d \sigma(u) \text { for every } x \in A .^{3}
$$

The problem of direct additivity of radial centre maps is discussed in [4].

If $\mathbf{s}=\left(s_{E}\right)_{E \in \mathcal{E}^{n}}$ is the family defined by (3.1) for a given sequence $\left(s^{(n)}\right)$ of selectors, then the corresponding families for extended selectors will be denoted by $\overline{\mathbf{s}}$ and $\tilde{\mathbf{s}}$.

We shall now prove that the extensions $s \mapsto \bar{s}$ and $s \mapsto \tilde{s}$ preserve direct additivity (Theorems 5.3 and 5.4). Let us start with the following.

Proposition 5.1. (i) For every subsets $A_{1}, A_{2}$ of $\mathrm{R}^{n}$

$$
\operatorname{ker}\left(A_{1}+A_{2}\right) \supset \operatorname{ker} A_{1}+\operatorname{ker} A_{2} .
$$

(ii) If $\mathrm{R}^{n}=E_{1} \oplus E_{2}$ (for $E_{1}, E_{2}$ non-necessarily orthogonal) and $A_{i} \subset E_{i}$ for $i=1,2$, then

$$
\operatorname{ker}\left(A_{1} \oplus A_{2}\right)=\operatorname{ker} A_{1} \oplus \operatorname{ker} A_{2} .
$$

Proof. (i) : Let $x_{i} \in \operatorname{ker} A_{i}$ for $i=1,2$ and $x=x_{1}+x_{2}$. Then for every $a \in A_{1}+A_{2}$ there exist $a_{i} \in A_{i}$ for $i=1,2$ such that $a=a_{1}+a_{2}$ and $\Delta\left(a_{i}, x_{i}\right) \subset A_{i}$. Since

$\forall t \in[0,1](1-t) x+t a=\left((1-t) x_{1}+t a_{1}\right)+\left((1-t) x_{2}+t a_{2}\right)$,

\footnotetext{
${ }^{2}$ On the p. 139 of [7] the restriction to $\mathcal{T}^{n}$ is not needed; $\mathcal{T}^{n}$ should be replaced by $\mathcal{S}_{0}^{n}$.

${ }^{3}$ In [7] this selector was denoted by $M_{\phi}$.
} 
it follows that

$$
\Delta(x, a) \subset \Delta\left(x_{1}, a_{1}\right)+\Delta\left(x_{2}, a_{2}\right) \subset A_{1}+A_{2} .
$$

Thus $x \in \operatorname{ker}\left(A_{1}+A_{2}\right)$.

(ii) : Let now $x \in \operatorname{ker}\left(A_{1} \oplus A_{2}\right)$. Then $x=x_{1}+x_{2}$ for a unique pair $\left(x_{1}, x_{2}\right) \in A_{1} \times A_{2}$. Take $a_{i} \in A_{i}$ for $i=1,2$ and let $a=a_{1}+a_{2}$; then $\Delta(x, a) \subset A_{1} \oplus A_{2}$, i.e.

$$
\forall t \in[0,1](1-t) x+t a \in A_{1} \oplus A_{2} .
$$

Thus $(1-t) x_{i}+t a_{i} \in A_{i}$ for every $t \in[0,1]$, whence $x_{i} \in \operatorname{ker} A_{i}$ for $i=1,2$. Therefore

$$
x \in \operatorname{ker} A_{1} \oplus \operatorname{ker} A_{2} .
$$

As a direct consequence of 5.1.(i), we obtain

Corollary 5.2. If $A_{1}$ and $A_{2}$ are star sets in $\mathrm{R}^{n}$, then so is $A_{1}+A_{2}$.

We shall now prove the following.

THEOREM 5.3. If $\mathbf{s}$ is a directly additive family of selectors for $\mathcal{K}_{0}^{n}$, then $\overline{\mathbf{s}}$ is a directly additive family of selectors for $\mathcal{S}_{0}^{n}$.

Proof. By $(5.1), \bar{s}^{(n)}(A)=\xi_{\text {ker } A}\left(s^{(n)}(\operatorname{conv} A)\right)$. Let $A=A_{1} \oplus A_{2}$ with $A_{i} \in \mathcal{K}\left(E_{i}\right)$ for $i=1,2$. Since $\mathbf{s}$ is directly additive and $\operatorname{conv} A=\operatorname{conv} A_{1} \oplus$ conv $A_{2}$, by Proposition 5.1(ii) combined with Lemma 2.2 it follows that

$\bar{s}^{(n)}(A)=\xi_{\text {ker } A_{1}}\left(s_{E_{1}}\left(\operatorname{conv} A_{1}\right)\right)+\xi_{\text {ker } A_{2}}\left(s_{E_{2}}\left(\operatorname{conv} A_{2}\right)\right)=\bar{s}_{E_{1}}\left(A_{1}\right)+\bar{s}_{E_{2}}\left(A_{2}\right)$.

By 3.3, Theorem 5.3 applies to the centroid, the Steiner point, and the Cebyšev point; by 4.4 , it applies to the $<\sigma_{0}>$-pseudo-centre:

Corollary 5.4. The families $\overline{\mathbf{c}}, \overline{\mathbf{p}}, \overline{\mathbf{c}}$, and $\overline{\mathbf{p}}_{<\sigma_{0}^{n}>}$ are directly additive.

Let us now pass to the second method of extending selectors from convex to star bodies.

ThEOREM 5.5. For every $n \geq 2$, let $s^{(n)}: \mathcal{K}_{0}^{n} \rightarrow \mathrm{R}^{n}$ be a selector satisfying condition (*). If for any $A_{i} \in \mathcal{K}_{0}\left(E_{i}\right)$ and $X_{i} \in \mathcal{K}\left(E_{i}\right)$ with $X_{i} \subset A_{i}$ for $i=1,2$

$$
s^{(n)}\left(A_{1} \oplus A_{2}, X_{1} \oplus X_{2}\right)=s_{E_{1}}\left(A_{1}, X_{1}\right)+s_{E_{2}}\left(A_{2}, X_{2}\right),
$$

then $\tilde{\mathbf{s}}$ is directly additive for $\mathcal{S}_{0}^{n}$.

Proof. Since $\operatorname{conv}\left(A_{1} \oplus A_{2}\right)=\operatorname{conv} A_{1} \oplus \operatorname{conv} A_{2}$ and, by 5.1(ii), $\operatorname{ker}\left(A_{1} \oplus\right.$ $\left.A_{2}\right)=\operatorname{ker} A_{1} \oplus \operatorname{ker} A_{2}$, it follows that for every $A_{i} \in \mathcal{S}_{0}\left(E_{i}\right), i=1,2$,

$$
\begin{aligned}
\tilde{s}^{(n)}\left(A_{1} \oplus A_{2}\right) & =s\left(\operatorname{conv}\left(A_{1} \oplus A_{2}\right), \operatorname{ker}\left(A_{1} \oplus A_{2}\right)\right) \\
& =s_{E_{1}}\left(\operatorname{conv} A_{1}, \operatorname{ker} A_{1}\right)+s_{E_{2}}\left(\operatorname{conv} A_{2}, \operatorname{ker} A_{2}\right) \\
& =\tilde{s}_{E_{1}}\left(A_{1}\right)+\tilde{s}_{E_{2}}\left(A_{2}\right) .
\end{aligned}
$$




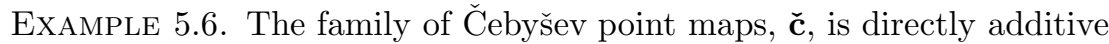
(see 3.3). Moreover, $\check{c}^{(n)}$ satisfies condition $(*)$; indeed, for every $A \in \mathcal{K}^{n}$, the point $\check{c}^{(n)}(A)$ is the unique minimizer of the function $\Psi_{A}: A \rightarrow \mathrm{R}$ defined by the formula

$$
\Psi_{A}(x):=\inf \{r>0 \mid B(x, r) \supset A\},
$$

thus $\check{c}^{(n)}$ satisfies the first part of $(*)$; it is easy to verify that it also satisfies the second part. Moreover, (5.4) holds. Hence $\tilde{\mathbf{c}}$ is directly additive.

EXAMPLE 5.7. By 4.4 combined with 5.5 , the family $\tilde{\mathbf{p}}_{<\sigma_{0}^{n}>}$ is directly additive, because the sequence of $\left\langle\sigma_{0}^{n}\right\rangle$-pseudo-centres satisfies condition $(*)$. Indeed, the first part of condition $(*)$ is obvious; it is also easy to prove the second part, because the proof of the uniqueness in [1] (compare Satz 1) is based on Hilfsatz 1, p.209, which can be applied in our situation as well.

\section{REFERENCES}

[1] I. Fáry and L. Rédei, Der zentralsymmetrische Kern und die zentralsymmetrische Hülle von konvexen Körpern, Math. Ann. 122 (1950), 205-220.

[2] H. Federer, Curvature measures, Trans. Amer. Math. Soc. 93 (1959), 418-481.

[3] R. Gardner, Geometric Tomography, Cambridge University Press, Cambridge, 1995.

[4] I. Herburt and M. Moszyńska, Remarks on radial centres for convex bodies, in preparation.

[5] E. Lutwak, Intersection bodies and dual mixed volumes, Advances in Math. 71(2) (1988), 232-261.

[6] M. Moszyńska, Remarks on the minimal rings of convex bodies, Studia Sci. Math. Hung. 35 (1999), 1-20.

[7] M. Moszyńska, Looking for selectors of star bodies, Geom. Dedicata 81 (2000), 131147.

[8] M. Moszyńska and T. Zukowski, On G-pseudo-centres of convex bodies, Glasnik Mat., 33(53) (1998), 251-265.

[9] M. Moszyńska and T.Żukowski, On G-pseudo-centres of convex bodies II, Supplemento al Rendiconti del Circolo Matematico di Palermo, Serie II, 70 (2002), 177-190.

[10] R. Schneider, On Steiner points of convex bodies, Israel J. Math. 9 (1971), 241-249.

[11] R. Schneider, Curvature Measures of Convex Bodies, Ann. Mat. Pura Appl. 116 (1978), 101-134.

[12] R. Schneider, Convex Bodies: the Brunn-Minkowski Theory, Cambridge University Press, Cambridge 1993.

Institute of Mathematics

Warsaw University

Banacha 2

02-097 Warszawa

Poland

E-mail: mariamos@mimuw.edu.pl

Received: 26.05.2003 